\title{
Optical Meteor Fluxes and Application to the 2015 Perseids
}

\author{
R. C. Blaauw, ${ }^{1 \star}$ M. Campbell-Brown, ${ }^{2}$ and A. Kingery ${ }^{3}$ \\ ${ }^{1}$ All Points Logistics/Jacobs ESSSA/NASA Meteoroid Environment Office, Huntsville, AL, USA, 35812 \\ ${ }^{2}$ University of Western Ontario, Department of Physics and Astronomy, London, ON, Canada, N6A 3K7 \\ ${ }^{3}$ ERC/Jacobs ESSSA/NASA Meteoroid Environment Office, Huntsville, AL, USA, 35812
}

\begin{abstract}
This paper outlines new methods to measure optical meteor fluxes for showers and sporadic sources. Many past approaches have found the collecting area of a detector at a fixed 100 $\mathrm{km}$ altitude, but this approach considers the full volume, finding the area in two km height intervals based on the position of the shower or sporadic source radiant and the population's velocity. Here, the stellar limiting magnitude is found every 10 minutes during clear periods and converted to a limiting meteor magnitude for the shower or sporadic source having fluxes measured, which is then converted to a limiting mass. The final output is a mass limited flux for meteor showers or sporadic sources. Presented are the results of these flux methods as applied to the 2015 Perseid meteor shower as seen by the Meteoroid Environment Office's eight wide-field cameras. The peak Perseid flux on the night of August 13, 2015, was measured to be 0.002989 meteoroids $/ \mathrm{km}^{2} / \mathrm{hr}$ down to 0.00051 grams, corresponding to a ZHR of 100.7 .
\end{abstract}

Key words: meteors - asteroids - comets

\section{INTRODUCTION}

The Solar System is filled with comets and asteroids which leave behind meteoroid streams as they orbit around the Sun. When Earth encounters one of these streams, it experiences a meteor shower. Many meteoroid streams have evolved over time due to planetary perturbations and non-gravitational forces in the Solar System, such as Poynting-Robertson drag, to become the sporadic background which accounts for the vast majority of meteoroids the Earth encounters. The sporadic background is not uniformly distributed, but originates from six main directions, called sporadic sources. The sporadic background has annual variation in its activity (CampbellBrown \& Jones 2006). Earth therefore sees variation in the meteoroid activity it encounters throughout the year both from the sporadic background and shower environments.

Finding the flux (number of particles per area per time) of these showers and sources is more useful than measuring rates, as it helps spacecraft designers and operators know how much protection to put on their spacecraft, and when to take precautions such as turning instruments away from a radiant. Fluxes can also better indicate true increases or decreases in activity, since they take into account the area of a detector, the radiant position of the population being studied, and other factors that affect how many meteors are seen.

Fluxes have been found in the past using radars, satellites, visual observations, lunar impact monitoring, or by studies such as Grun et al. (1985) which used zodiacal light photometry, spacecraft measurements, and lunar microcraters to obtain fluxes over a wide mass range. Each detector or method has advantages and disadvan-

^ E-mail: rhiannon.c.blaauw@nasa.gov tages, a primary one being the size range over which detector can measure fluxes. Patrol radars, like the Canadian Meteor Orbit Radar which measures meteor fluxes in the 100 micron size-range, have the advantage of detecting meteors in the daylight and through clouds and thereby obtaining fluxes for daytime showers, but suffer from the initial trail radius problem which makes it difficult for them to detect faster showers (Campbell-Brown et al. 2006). In situ detectors, even though limited by small collecting areas and to very small particles, have the advantage of not using light or ionized proxies to infer meteoroid properties. They are also able to determine fluxes at distances other than $1 \mathrm{AU}$ by sending spacecraft to different places in the Solar System, such as the ESA/NASA mission Ulysses and the NASA spacecraft Galileo which flew by Jupiter in 1992 (Landgraf et al. 2000). Lunar impact monitoring provides a large collecting area by using the surface of the Moon to detect flashes from large meteoroids impacting the Moon, but is constrained by the phase of the Moon and the weather (Suggs et al. 2014).

Video observation of meteors is one of the youngest and most rapidly progressing observing techniques in meteor science. It has particularly excelled when the detection and analysis routines have been automated. Video observations cover everything from fireball detections of $\mathrm{cm}$-sized meteoroids using all-sky cameras, to intensified cameras detecting 100 micron meteoroids using a smaller field of view. Thus optical detectors can enable fluxes to be determined over a wide range of masses.

Optical meteor fluxes have been published in the past, but this study sets out to look at the problem in a more thorough way. The approach considers the full volume, finding the area in $2 \mathrm{~km}$ altitude increments and using the area at the true meteor height, not assuming an average height such as $100 \mathrm{~km}$ which past studies have 
done (Greenhow \& Hall 1960). The area can be substantially different from $60 \mathrm{~km}$ to $140 \mathrm{~km}$, the range of heights over which most meteoroids ablate, thus having a single collecting area is an oversimplification. This approach also finds the limiting absolute meteor magnitude at regular time intervals throughout a clear period using the limiting stellar magnitude, camera characteristics, and radiant and speed of the shower or source. This is a significant improvement over estimating a single limiting magnitude over the course of the night based on the magnitude distribution of the meteors detected, and takes into account ever-changing sky conditions, the movement of the radiant over the night, and the change in movement of the meteor over the field of view.

\section{EQUIPMENT}

The algorithms presented here are currently implemented on eight wide-field meteor cameras operated by NASA's Meteoroid Environment Office, producing shower and sporadic source fluxes.

The cameras are located at two sites $31.7 \mathrm{~km}$ apart, four being in Huntsville, Alabama, and four in Decatur, Alabama. Each of the eight cameras consist of a Watec 902H2 Ultimate CCD video camera, four with a $17 \mathrm{~mm}$ focal length Schneider lens (f/0.95), and four with a $17 \mathrm{~mm}$ focal length Navitar lens (f/0.95), each producing a $22 \times 16$ degree field of view. Pairs of cameras, one at each site, are pointed at overlapping volumes of the atmosphere. Two sets of cameras point roughly north, and two sets point roughly south. This configuration detects up to 100 meteors down to a magnitude of +5 on a non-shower night. The methods in this paper are applied to this system, but can be translated to any optical system. This paper presents the results of the 2015 Perseid meteor shower as seen from these wide-field meteor cameras.

\section{METEOR DETECTION AND SHOWER IDENTIFICATION}

Video from the cameras is processed with the All Sky and Guided Automatic Real-time Detection (ASGARD) (Weryk et al. 2008) software which performs the meteor detection and photometry, and invokes the MILIG and MORB (Borovicka 1990) codes to determine the trajectory, speed, and orbit of each meteor. A meteor is identified as belonging to a shower if its radiant comes within 7.5 degrees of the shower radiant, and its velocity is within $20 \%$ of the shower velocity.

Since the field-of-view of the cameras used in this study is small enough that meteors are often detected entering and/or leaving the field-of-view, one needs to decide which meteors will be counted in the flux determinations. The majority ( $78 \%$ of the 12,385 meteors seen in 2015) of the meteors are seen starting and ending in the fieldof-view of the cameras, so this is a minor effect for many shower and sporadic sources; however for faster meteors and radiants far from the camera pointing, more partial trails are detected and the question of which meteors should be counted is more significant. Since this study defines the height of the meteor as the height of its brightest point (see Section 4.1 Collecting Area, below) and a peak magnitude and corresponding mass can be found, a meteor is used for fluxes if its brightest point occurs in the volume. If there exists a noticeable increase and decrease in the brightness of the meteor in the part of its trail detected (a peak magnitude is seen), it is considered in the volume. If the meteor is only seen increasing in brightness or decreasing in brightness before leaving the field-of-view, the peak brightness of a meteor is considered likely outside of the volume and it is not counted in flux calculations. This of course does not properly account for meteors that have double peaks or irregular light-curves. Given that the number of partial trails is small, this is a reasonable compromise between including all meteors and excluding all meteors with partial trails.

A detection efficiency factor is needed to account for imperfect detection. In post-processing routines, the false detections (categorized as bugs, birds, clouds or other objects instead of meteors) are scanned for missed meteors in the original detection. Video was visually scanned to ensure that all meteors seen in the video were either caught by the original detection, or recovered from false detections.

\section{FLUX METHODOLOGY}

To derive accurate optical meteor fluxes one needs to know both the effective area and the limiting magnitude (which would then correspond to a limiting mass) for the detector for each active shower and sporadic source.

Section 4.1 describes the collecting area of the detector and the corrections applied to it, Section 4.2 discusses how the limiting stellar magnitude of an image is found, Section 4.3 covers how to convert the limiting stellar magnitude to a limiting meteor magnitude, and Section 4.4 explains the conversion from magnitude to mass in order to find a limiting mass.

\subsection{Collecting Area}

The collecting area is defined as the physical area of the sky over which the optical systems can detect meteors from a particular meteor shower or sporadic source. There are two main steps in finding the collecting area. First, the raw area of the sky that can be seen at each height is found, then corrections are applied to find the effective collecting area for a particular meteor shower or sporadic source; the collecting area used in flux calculations. These corrections are to account for the meteors we do not see because of: the range to the meteors (the brightness of meteors fall off as the square of their range), the angular velocity of the meteors (the spreading out of light makes it more difficult to detect meteors), the elevation of the radiant (projection of the radiant on the visible sky), the camera hardware (lower sensitivity in some pixels than others), and the camera software.

The collecting areas used in this work are found as a function of height, in $2 \mathrm{~km}$ intervals. This is a significant difference from past meteor flux work which assumes a single height, usually 100 $\mathrm{km}$ or an average height based on the velocity, to find the collecting area (Molau \& Barentsen 2014, 2013; Ott et al. 2014). The height of a meteor is taken to be the height of its brightest point. When a single height is assumed (e.g. $100 \mathrm{~km}$ ) to find the collecting area, the number of meteors seen in the volume is divided by that area at $100 \mathrm{~km}$. When an area for the specific height is used, each meteor is divided by the collecting area at its height and summed to find the total flux.

The raw area in a multistation volume is found by generating a three-dimensional grid of blocks between specified heights, and identifying which of those blocks can be seen by an individual camera and by two cameras combined. The size of the blocks is determined by the size of the field-of-view of the camera, smaller blocks being more appropriate for smaller fields-of-view. For cameras wider than 20 degrees (used in this study), 4 x $4 \mathrm{~km}$ bins were 


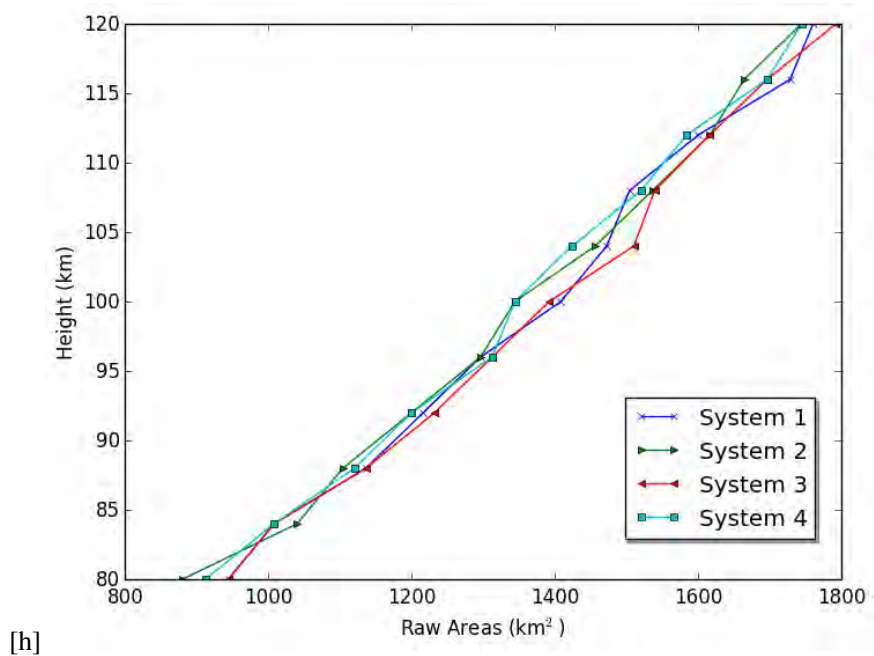

Figure 1. Raw collecting areas seen by each two-station system, in square kilometers, given every 2 kilometers from 80 to 120 kilometers.

found to be suitable, as smaller bins did not result in marked improvements. The block dimensions are the same at all heights, but the number of blocks is different between heights due to the change in collecting area. See Figure 1 for raw collecting areas for the wide-field cameras in north Alabama. Once the raw area is found, corrections are applied to determine the effective collecting area.

The sensitivity of the camera is applied to the collecting area using the flat field. A flat field is taken for the camera and normalized to the brightest pixel. Each block of the collecting area is observed with a particular pixel of the camera (or multiple pixels, in which case the center pixel is used). The sensitivity of the camera at that pixel is multiplied with the raw area of the block. This is necessary as most optical systems do not have uniform sensitivity across their field of view; for example, most have vignetting near their edges and are more sensitive in the middle than at the edge.

The angular speed of the meteor is also taken into account in the collecting area. The angular speed of a meteor from a particular radiant at a particular speed is found for each block, and then normalized to the angular velocity and range at the center of the field of view. This is to account for a difference in detection across the field of view due to the angular velocity, with larger angular speeds leading to lower brightness, and is explained more thoroughly in Section 4.3 Limiting Meteor Magnitude.

The projection angle of the meteor shower radiant is multiplied to the collecting area by the cosine of the zenith angle. When the radiant is directly overhead, the maximum collecting area is seen. When the radiant is sitting just above the horizon, the effective collecting area is much lower. This is due to the stretching of the light across the sky depending on the position of the radiant. This is different from the angular velocity correction which depends on where a system is looking. The projection angle correction is only affected by the elevation of the radiant. This correction can be applied to the fluxes at any point; for convenience we choose to apply it to the collecting areas.

The range from the camera to a block affects the detection efficiency of the meteors as the brightness of meteors falls off as the square of their range. However, using meteor absolute magnitude accounts for range and sets the range at $100 \mathrm{~km}$. Therefore range correction is scaled to $100 \mathrm{~km}$. Meteors with the same magnitude will be less likely observed further than $100 \mathrm{~km}$ than they would

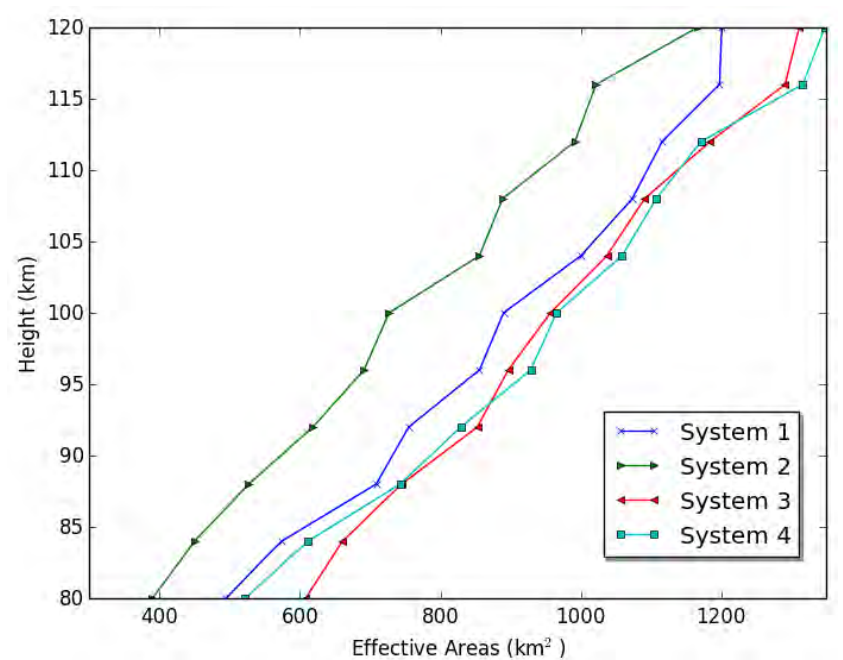

Figure 2. Effective Perseid collecting areas seen by each two-station system, in square kilometers, given every 2 kilometers from 80 to 120 kilometers.

be closer than $100 \mathrm{~km}$. Or another way to say this is that we will observe dimmer meteors closer than $100 \mathrm{~km}$ than we will further than $100 \mathrm{~km}$ and this needs to be accounted for.Additionally, our approach tries to separate the physical area over which meteors can be detected, from the limiting brightness of a meteor that can be detected in that physical area, and the range to each of the collecting area blocks affects the limiting magnitude of the meteors that can be seen at each block.

Each of these corrections must be raised to $s-1$, s being the mass index. The mass index indicates the distribution of mass in the meteoroid stream; a mass index less than 2 means there is more mass in larger particles, a mass index of 2 means the mass is evenly distributed, and a mass index more than 2 means more mass is contained in smaller particles. The corrections made to the raw area to produce the effective area account for the meteors not seen, though to know accurately how many meteors may not be detected, one needs to know the distribution of masses in the stream. If the mass index is not known, a larger uncertainty is introduced into the collecting areas and fluxes. The choice is between assuming whether there is more mass in smaller or larger particles, which will introduce errors, or leaving the mass index at 2 (equal mass distribution across bins). This approach leaves the mass index at 2 when it is unknown, such as for new or poorly observed showers.

When these corrections are applied we have the effective collecting area - the area corresponding to the faintest meteor from the shower or sporadic source which can be seen at the center of the field of view at a range of $100 \mathrm{~km}$. The limiting magnitude should be representative of this meteor - the faintest meteor seen at the center of the field of view. This area is then applied directly to rates of meteors to obtain fluxes. Figure 2 contains the effective collecting areas of Perseid meteor shower on 2015-08-13 at 7:00 UTC for those same cameras.

\subsection{Limiting Stellar Magnitude}

The stellar limiting magnitude is the faintest star for which the system under particular sky conditions will detect all stars of equal or greater brightness. This value will depend on the camera hardware, as well as sky conditions. The limiting stellar magnitude is used 

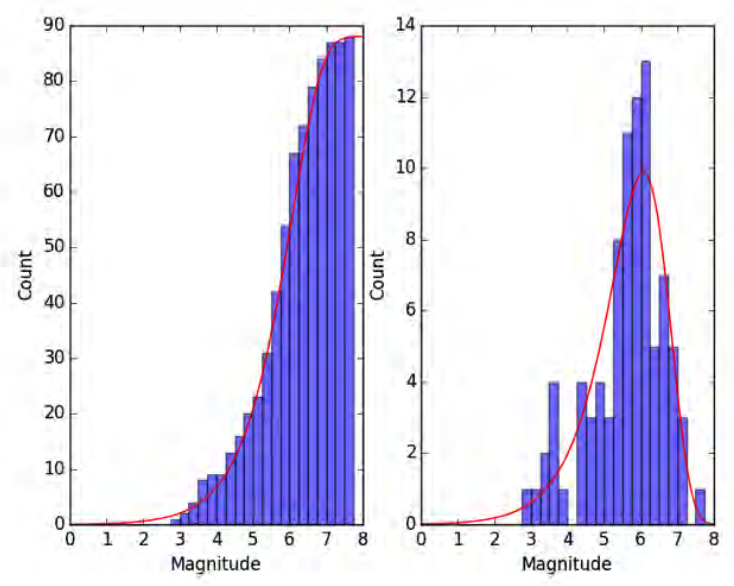

Figure 3. Counts versus R-magnitude of all stars identified in the field of view of a calibration image. On the left is a cumulative distribution and on the right the probability density. The limiting magnitude is the mode of the fit distribution.

to find the limiting absolute meteor magnitude, which gives us a limiting mass for the flux values.

The limiting stellar magnitude is found by employing astrometry.net and the Python package Astropy (Greenfield et al. 2014) to determine the positions of stars that should be able to be identified in the field-of-view of the image at a specified time. If a star is distinguishable above the background, its signal-to-noise ratio (SNR) is calculated using aperture photometry. A threshold SNR of five was chosen as it was the best value which caught the dimmest stars in the field of view, but did not flag noise as stars. Combining the known magnitudes and measured SNR of many stars in the field of view, the magnitude of stars with a SNR above the background are fit to a Gumbel distribution. The limiting magnitude is taken to be the mode of the distribution. See Figure 3.

The limiting stellar magnitude should be found at regular intervals throughout the observing period, as the sky conditions can change. No times with partial cloudy images are used. Because the fields-of-view are not very large, this is not a significant fraction of the data. The limiting stellar magnitude is between +6 and +7 for a clear time period for the wide-field systems discussed here. The limiting stellar magnitude is measured for every 10 minute clear period using 40 second stacked video images. It is important to monitor how the limiting magnitude changes throughout the night, as sky conditions can quickly change, and a 0.5 magnitude uncertainty in limiting magnitude translates to an uncertainty in limiting mass by a factor of two. See Figure 4 for the limiting stellar magnitude of the eight wide-field cameras over the night of 2015-08-13.

\subsection{Limiting Meteor Magnitude}

The limiting absolute meteor magnitude is defined as the faintest absolute meteor magnitude for which a system will detect all meteors of greater or equal brightness. The limiting absolute magnitude is necessary to find the limiting mass corresponding to the measured fluxes. The relationship between the limiting stellar magnitude and limiting apparent meteor magnitude is determined by the camera hardware, software, and angular velocity of the meteor. The difference in apparent to absolute magnitude is caused by the range.

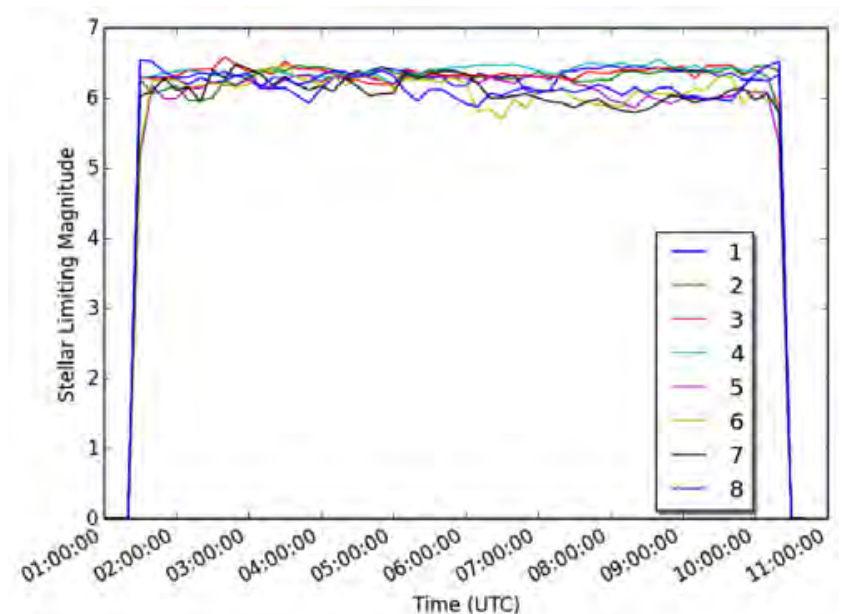

Figure 4. Limiting stellar magnitudes for each of the eight wide-field meteor cameras on 2015-08-13.

The conversion from stellar to meteor magnitude accounts for the movement of the meteor spreading its light out over multiple pixels. This movement makes the meteor fainter than a star of equivalent brightness, in which the light is concentrated over a few pixels. Explicitly this relationship is:

$m_{M}=m_{s}-2.5 \log \left(\frac{d}{f w h m}\right)$

$d=\left(\frac{180 r_{1} V \tau \sin \zeta}{\pi F_{O V} R}\right)$

where $m_{M}$ is the apparent meteor magnitude, $m_{S}$ is the apparent stellar magnitude, and $d$ is defined as the distance moved by the meteor (in pixels) during a single video frame, determined by the speed and radiant of the meteor, as well as the camera specifications. The resolution of the detector in number of video lines is $r_{1}, V$ is the geocentric velocity in meters/second, $\tau$ is the effective CCD integration time, $\zeta$ is the angle between the radiant and pointing direction of the camera, $F_{O V}$ is the angular size of the field of view, $f w h m$ is the point-spread function for the camera (full width half $\max$ ), and $R$ is the range to the meteors (Brown et al. 2002; Hawkes 1993) Combining equations (1) and (2) with the conversion from apparent to absolute magnitude results in:

$M_{M}=m_{s}-5 \log \left(\frac{R}{100 k m}\right)-2.5 \log \left(\frac{180 r_{1} V \tau \sin \zeta}{\pi F_{O V} R(f w h m)}\right)$

where $M_{M}$ is the absolute meteor limiting magnitude.

The difficulties with finding the absolute limiting magnitude from the stellar magnitude using this equation are that it requires a single point in the field of view and single range to calculate the angular velocity correction. This assumes that the angular velocity correction is the same across the field, when in fact the field of view covers many azimuths and elevations, and a single azimuth and elevation can cover various ranges. This is not a significant problem when the radiant is far from the field of view and therefore at similar distances from any point in the field of view (or when the field is very small). However it becomes more of a problem when the radiant is very close to the camera pointing angle, causing the angular velocity of meteors to be significantly different on opposite 
sides of the field of view, thus leading to different limiting meteor magnitudes across the field.

One can apply this angular velocity correction in two places; the collecting area or the limiting meteor magnitude. This most intuitively affects the limiting magnitude, since the faster the meteor, the more the light will be spread out and the brighter the meteor needs to be to reach the detection limit, but it can also be viewed to change the effective collecting area (making it larger if a meteor is more likely to be detected, or smaller if a meteor is less likely to be detected). Applying the angular velocity correction to either the collecting area or limiting magnitude has the same effect on the end flux results. However, since a single absolute meteor magnitude is needed that can give us the limiting mass of our fluxes at that time, it is best to apply the correction in two steps. A single absolute limiting meteor magnitude is found using Equation (3) at the center of the field-of-view and at an average range that the meteors are detected, based on the camera pointing (for the wide-field cameras discussed here that range is $130 \mathrm{~km}$ ). An angular velocity correction is also found for each block of the collecting area, and is then normalized to the angular velocity correction at the center of the field of view, and the average range. This approach gives an absolute limiting magnitude for each system at each time, yet has each segment of the volume corrected for the angular velocity effect on detection. The limiting meteor magnitude is typically 1-3 magnitudes brighter than the limiting stellar magnitude. See Figure 5 for the limiting absolute meteor magnitude for the Perseid meteor shower in the eight widefield cameras used in this study, for the night of 2015-08-13. Note in this example, the Perseid radiant came very close to cameras 2 , 3 and 4 which produces dimmer limiting magnitudes due to the light being less spread out from the angular velocity of the meteor. Meteors are be detected that are fainter than the limiting magnitude, as the limiting magnitude only indicates the completeness limit, not an absolute limit, however those meteors are not included in flux calculations.

Another thing to note about Equations (1) to (3) is that when $d$ is less than $f w h m$, the meteor magnitude can become larger than the stellar magnitude. But this is not physical since the formula describes the loss in limiting magnitude from the motion of the meteor. Thus in this rare case, $d$ is set to $f w h m$ and the meteor limiting magnitude equals that of the stellar limiting magnitude which is expected since the meteor would be a point-source.

The limiting meteor magnitude is found for every 10 minute clear period. Fluxes for the wide-field cameras are given to the average limiting magnitude over the course of the night. If the limiting magnitude varied throughout the night, the fluxes found in that individual time period are scaled using their population index to the average limiting magnitude. This is done to eliminate the overall error from scaling. Scaling a flux to a much higher or lower limiting magnitude includes assumptions about its population, which can introduce significant errors, thus scaling each individual flux to the average limiting magnitude introduces the least amount of error.

\subsection{Magnitude to Mass Conversion}

A limiting mass for the fluxes can be found from the limiting absolute meteor magnitude. This study uses the relationship derived by Brown, found in Peterson (1999) to convert magnitude to mass:

$M=-8.75 \log \left(v_{\text {inf }}\right)-2.25 \log (m)+11.59$

where $M$ is peak absolute magnitude, $m$ is mass (grams) and $v_{\text {inf }}$ is the approach velocity (kilometers/second). A limiting meteoroid

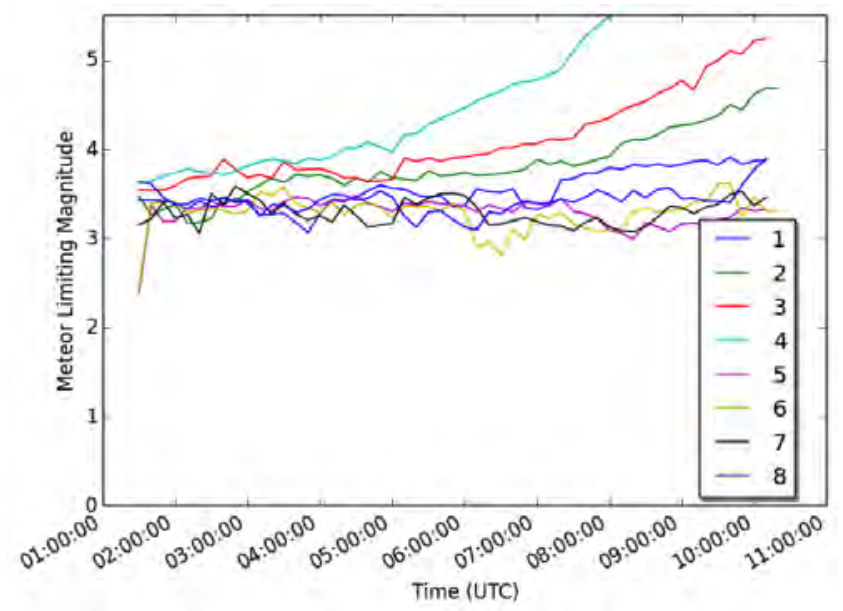

Figure 5. Perseid limiting absolute meteor magnitudes for each of the eight wide-field meteor cameras on 2015-08-13.

mass is calculated for the average absolute limiting meteor magnitude for every active sporadic source and shower. Converting from magnitude and velocity to mass is not trivial as the luminous efficiency (percentage of kinetic energy converted to light) is a highly uncertain quantity and is difficult to measure. See Weryk \& Brown (2013) for a summary of luminous efficiency values found using a variety of methods.

Meteoroid fluxes are particularly useful for quantifying spacecraft risk. They can be used to determine how many meteors from a specific shower or source can be expected to impact a spacecraft, but energy of the collision depends on the mass, thus knowing the limiting mass over which the fluxes are effective is important. One of the largest areas of uncertainty in the flux is likely the conversion from magnitude to mass. Uncertainties in the luminous efficiency can result in uncertainties in masses up to an order of magnitude.

\section{RESULTS}

The methods presented here are applied to observations of the 2015 Perseid meteor shower, from August 9 through August 14, 2015 by the Meteoroid Environment Office's wide-field meteor cameras. Perseid fluxes are difficult to calculate using radars because, being a fast shower, they are strongly affected by initial trail radius attenuation (Campbell 2002). Thus optical or visual observations of Perseids often prove more effective than radar observations. These cameras saw 223 double-station Perseids in 2015; 92 of those occurred on the peak night of August 13. Table 1 contains a summary of the results. Starting on August 15, Alabama had several cloudy nights, permitting fluxes to be calculated only through August 14 .

Figure 6 has the fluxes scaled to the average limiting magnitude, and Figure 7 shows the Perseid Zenithal Hourly Rate (ZHR) found each night through its activity. The ZHR of a shower is commonly used to measure the strength of its activity and indicates how many visual meteors can be seen in clear conditions when the radiant is at the zenith. The ZHR is calculated from the flux at a limiting magnitude of +6.5 and uses the population index to extrapolate the activity to magnitudes that would be seen by a visual observer (Koschack \& Rendtel 1990b). As fluxes are often output to +6.5 for ease of comparison, they are included here in Table 3, but one should note that these fluxes are extrapolated from the val- 
Table 1. Fluxes, limiting magnitudes, limiting masses, and ZHRs for the 2015 Perseid meteor shower as soon by the Meteoroid Environment Office's eight wide-field cameras.

\begin{tabular}{|c|c|c|c|c|c|}
\hline Date & Flux $\left(10^{-4}\right.$ meteors $\left./ \mathrm{km}^{2} / \mathrm{hr}\right)$ & Error $\left(10^{-4}\right.$ meteor $\left.\mathrm{s} / \mathrm{km}^{2} / \mathrm{hr}\right)$ & Limiting Magnitude & Limiting Mass $\left(10^{-4}\right.$ grams $)$ & ZHR \\
\hline 20150809 & 2.86 & 1.91 & 3.5 & 5.1 & 10.0 \\
\hline 20150810 & 2.73 & 1.73 & 3.5 & 5.1 & 9.7 \\
\hline 20150811 & 2.71 & 1.92 & 3.3 & 6.3 & 11.3 \\
\hline 20150812 & 6.71 & 3.16 & 3.5 & 5.1 & 23.0 \\
\hline 20150813 & 29.89 & 6.86 & 3.5 & 5.1 & 100.7 \\
\hline 20150814 & 12.80 & 7.10 & 3.4 & 5.7 & 48.1 \\
\hline
\end{tabular}

ues found at their intrinsic limiting magnitudes to +6.5 using the assumed population index. The population index is derived from the mass index using Equation 5. The ZHR is then dependent on the population index both in scaling fluxes down to +6.5 and then scaling up to visual rates. An incorrect population index can introduce significant uncertainties here. Figure 7 also has the clear hours over the camera locations per night plotted alongside the ZHRs. The number of clear hours is taken into account when finding fluxes.

$s=1+2.3 \log (r)$

All scaling and ZHRs in this study are found assuming a mass index of 1.8. This mass index is taken from Hughes (1973) in which a mass index of 1.78+/- 0.04 is found for the Perseids, from Brown (1999) where mass indices of 1.61 to 1.93 were found for the 1988 to 1994 Perseids, and from Arlt \& Händel (2000) who found a mass index of between 1.8 and 1.95 for the peak of the 2000 Perseids. The flux methods were developed to depend on the mass index as little as possible, outputting fluxes to an average limiting magnitude per night so that each flux value throughout the night is scaled as minimally as possible. Table 2 shows how much the mass index affects the overall flux value, showing the fluxes found with mass indices of 1.6 to 2.2. Note that the mass index has an effect on the flux value and the ZHR in different ways. A larger mass index indicates more mass in smaller particles and since the mass index corrects for what we do not see, this produces an effect of increasing the flux. But note that the mass index is also used in the expression to go from flux to ZHR, which is an indication of visual rates (caused by larger particles). Thus a smaller mass index (indicating a greater amount of mass in more massive particles) will create a larger ZHR. If mass indices of 1.6 and 2.0 are both used to calculate ZHRs on the exact same flux value +6.5 magnitude, the ZHR will be larger when using a mass index of 1.6.

The results presented here match past Perseid ZHR profiles well, for example the 2013 and 2014 Perseid profiles created by the International Meteor Organization based on visual observations. The Perseids are frequently seen to have ZHRs of 5-15 two weeks prior to the peak, 15-25 the week before the peak, and peak ZHRs between 100 and 120 (Olech 2000; Arlt \& Händel 2000; Rendtel \& Brown 1997).

The average height of the Perseids was $104 \mathrm{~km}$. If the fluxes were found assuming a $100 \mathrm{~km}$ altitude and a collecting area corresponding to $100 \mathrm{~km}$, this flux would be determined to be a $9 \%$ increase in flux over that found using the true height of the meteor. The difference is relatively small in this case, as there is only a $4 \mathrm{~km}$ difference; however many showers ablate at $90 \mathrm{~km}$ or below. The May Camelopardalid meteor shower outburst in May, 2014 was observed to have average heights of $81 \mathrm{~km}$ using these same wide-field cameras. The collecting area at $100 \mathrm{~km}$ is $71 \%$ greater than at the

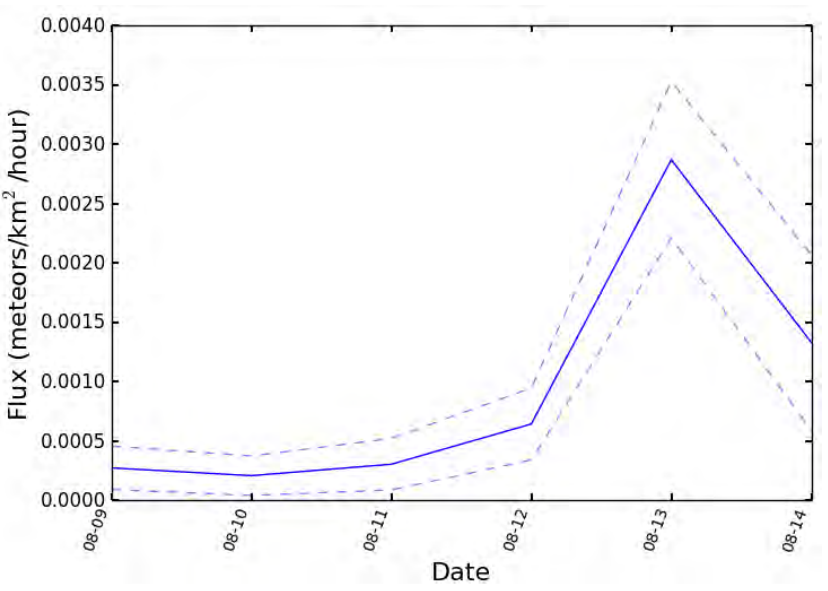

Figure 6. Perseid fluxes to a limiting magnitude of 3.45 which corresponds to a limiting mass of $5.4 \mathrm{e}-4$ grams.

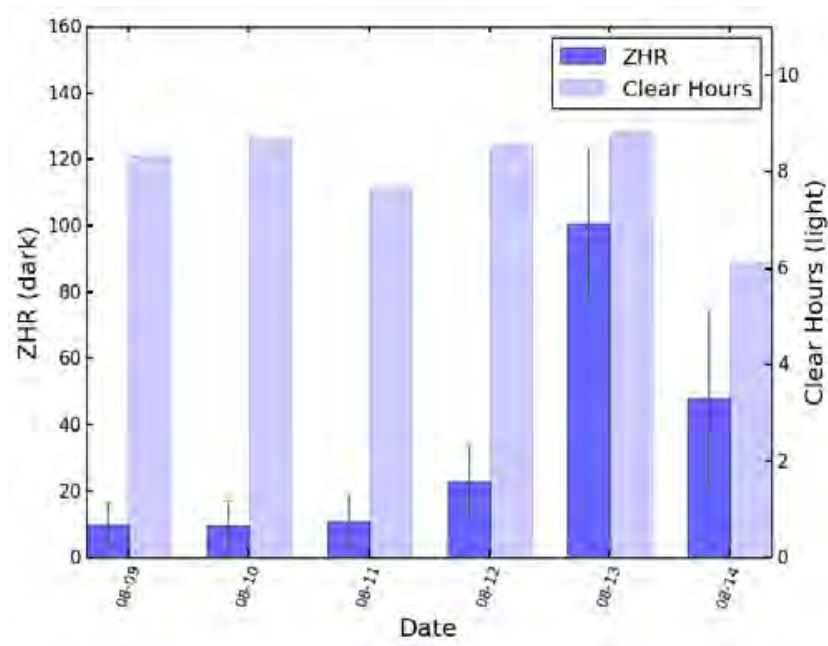

Figure 7. 2015 Perseid ZHRs and clear observing hours per night.

$81 \mathrm{~km}$. Thus assuming a $100 \mathrm{~km}$ height would lead to larger errors in the flux for the May Camelopardalids. 
Table 2. Perseid fluxes and ZHRs on 2015-08-13 down to +3.5 magnitude when using different mass indices.

\begin{tabular}{lcc}
\hline Mass Index & Flux $\left(10^{-3}\right.$ meteors $\left./ \mathrm{km}^{2} / \mathrm{hr}\right)$ & ZHR \\
\hline 1.6 & 2.549 & 124.2 \\
1.8 & 2.989 & 100.7 \\
2.0 & 3.535 & 104.3 \\
2.2 & 4.215 & 122.9 \\
\hline
\end{tabular}

Table 3. Perseid fluxes to a limiting magnitude of +6.5 .

\begin{tabular}{lc}
\hline Date & Flux $\left(10^{-3}\right.$ meteors $\left./ \mathrm{km}^{2} / \mathrm{hr}\right)$ \\
\hline 20150809 & 3.24 \\
20150810 & 3.14 \\
20150811 & 3.64 \\
20150812 & 7.44 \\
20150813 & 32.58 \\
20150814 & 15.56 \\
\hline
\end{tabular}

\section{DISCUSSION AND CONCLUSIONS}

Presented here are new methods to find rigorous optical meteor fluxes for showers and sporadic sources. The methods described are applied automatically every morning on eight wide-field cameras operated by NASA's Meteoroid Environment Office.

Several approaches to optical meteor fluxes have been tried in the past (Molau \& Barentsen 2014; Ott et al. 2014) as well as meteor fluxes from visual observations (Koschack \& Rendtel 1990b,a) and radar (Brown \& Jones 1995; Campbell-Brown et al. 2006). The routines and procedures used here are different than others in use in several ways. These routines use double-station data in which orbits and trajectories have been found, more accurately identifying which meteors belong to a shower than using single-station data, in which a meteor can only be approximately associated with a shower. These routines also do not assume a uniform $100 \mathrm{~km}$ height for the collecting area, nor a single set height based on velocity and zenith angle. Instead, the collecting area is found per height in $2 \mathrm{~km}$ bins within the common volume observed by the cameras and the area at the height of the meteor is used. Because of the difference in area between 80 and $120 \mathrm{~km}$ for an optical detector, finding the collecting area at the height at which a meteor ablates instead of assuming a single height should be considerably more accurate. Choosing a fixed height based on velocity would eliminate a scatter in heights from the size of the meteoroid (larger particles penetrate deeper); however it would introduce a bias since meteoroids with the same speed but different composition tend to ablate at different heights (Jenniskens et al. 2016).

The methods here are also different from other optical flux methods in that they find a limiting stellar magnitude at regular intervals during any clear period (this study uses every 10 minutes), which accounts for varying sky conditions, and a limiting meteor magnitude for every shower or source for each limiting stellar magnitude. This limiting magnitude takes into account the difference in angular velocity over the entire volume where a meteor can be observed. Often flux studies assume a single limiting magnitude, using the magnitude distribution of the meteors observed to find the rollover magnitude and estimate a limiting magnitude over the whole observation period. This is an oversimplification since the limiting meteor magnitude most certainly changes throughout the night even if the sky conditions stay the same, because the position of the radiant changes, leading to a change in the angular velocity of the meteors. Molau and Barentsen are also an exception to this, as they find a limiting magnitude every minute for the IMO Video Meteor Network fluxes. Instead of finding the R-magnitude cutoff at which stars are no longer seen, their approach entails counting the total number of stars seen in the field-of-view, and deriving the limiting magnitude based on the number of identified stars (Molau $\&$ Barentsen 2013, 2014). Their approach and the approach presented in this paper allow fluxes to be found over a short period of time that may not have the number statistics to find a good limiting magnitude using only the meteors' magnitude distributions. These fluxes with small number statistics will have large uncertainties, but they can still be helpful and indicative of the activity (or lack of activity) of a meteor shower.

The fluxes produced by these algorithms give separate fluxes for sporadic sources, instead of a single sporadic flux, as presented by past studies (Kretschmer et al. 2015). This is important for two reasons. First, the collecting area and limiting magnitude can be quite different for each source since the radiant and speed are not uniform for sporadic meteors. The apex sources have velocities around $60 \mathrm{~km} / \mathrm{s}$ whereas the antihelion and north toroidal source are much slower with velocities of $30-35 \mathrm{~km} / \mathrm{s}$ (Campbell-Brown et al. 2006). A second benefit to separating sources instead of finding one sporadic flux is finding variation throughout the year for sporadic sources. According to Campbell-Brown et al. (2006), the flux of sporadic sources varies significantly throughout the year, and varies at different levels and different times for each source. Optical flux routines such as those discussed in this paper will allow a study on flux variation in a size range above that of radar.

Though radars have the advantage of being able to observe during the day and through clouds, they suffer different biases than optical systems, especially for faster meteor showers which suffer from the initial trail radius effect, thus optical fluxes can help calibrate radar fluxes for fast showers (Campbell 2002).

Optical fluxes fill in the gap between fluxes that are found on a large scale such as fluxes from lunar impact monitoring (Suggs et al. 2014) or infrasound (Brown et al. 2013), and fluxes found on a very small scale such as in situ data from satellites (Landgraf et al. 2000). Optical fluxes will allow many intermediate flux values as optical systems can detect meteors from 100 micron to $\mathrm{cm}$ sized meteoroids. The fluxes found with in situ data, infrasound measurements of bolides, or lunar impact data requires long time periods to either collect the data or obtain the number statistics necessary for meaningful fluxes. Since video systems are able to be automated, fluxes can be calculated quickly. This quick turnaround allows measurements of meteor shower outbursts and flux measurements on small time-scales which cannot be found with in situ observations for the smallest particles or bolide data for the largest particles.

These routines allow flux measurements in many size ranges as optical systems cover a wide range of meteoroid observations. Deriving fluxes in various mass-ranges is important because it eliminates using highly uncertain mass indices to extrapolate fluxes to different masses. Additionally, finding fluxes to different masses can improve mass indices and allows a determination of mass indices over larger ranges.

There is still work that can be done on improving optical meteor fluxes. It will be helpful to characterize the effect of the radiant altitude on the ability to detect meteors, which is applied in the collecting area. This is usually taken to be the cosine of the zenith angle but this simple dependence has been questioned (Molau \& Barentsen 2013). A study of more well-characterized showers (like 
the Geminids) would further test these methods, as well as studies of one shower detected in several optical systems down to different limiting magnitudes.

Meteor fluxes found with optical instruments are of great practical and scientific interest. This study, with future work, will help constrain our understanding of both the sporadic and shower meteoroid environment and may lead to new insights about meteoroid streams and sporadic sources, indicating variation throughout the stream.

\section{ACKNOWLEDGEMENTS}

The authors would like to thank Dr. William J. Cooke, Dr. Robert M. Suggs, and Dr. Peter G. Brown for the many discussions regarding optical meteor fluxes. This work was supported in part by NASA Cooperative Agreement NNX15AC94A (MCB) and NASA contract NNM12AA41C (RCB and AK).

\section{REFERENCES}

Arlt R., Händel I., 2000, WGN, Journal of the International Meteor Organization, 28, 166

Borovicka J., 1990, Bulletin of the Astronomical Institutes of Czechoslovakia, 41,391

Brown P. G., 1999, PhD thesis, THE UNIVERSITY OF WESTERN ONTARIO (CANADA)

Brown P., Jones J., 1995, Earth Moon and Planets, 68, 223

Brown P., Campbell M., Suggs R., Cooke W., Theijsmeijer C., Hawkes R. L., Jones J., Ellis K. J., 2002, MNRAS, 335, 473

Brown P. G., et al., 2013, Nature, 503, 238

Campbell M. D., 2002, PhD thesis, THE UNIVERSITY OF WESTERN ONTARIO (CANADA

Campbell-Brown M. D., Jones J., 2006, MNRAS, 367, 709

Campbell-Brown M., Vaubaillon J., Brown P., Weryk R. J., Arlt R., 2006, A\&A, 451, 339

Greenfield P., Tollerud E. J., Robitaille T., Developers A., 2014, in American Astronomical Society Meeting Abstracts \#223. p. 255.24

Greenhow J. S., Hall J. E., 1960, MNRAS, 121, 174

Grun E., Zook H. A., Fechtig H., Giese R. H., 1985, Icarus, 62, 244

Hawkes R. L., 1993, in Stohl J., Williams I. P., eds, Meteoroids and their Parent Bodies. p. 227

Hughes D. W., 1973, MNRAS, 161, 113

Jenniskens P., et al., 2016, Icarus, 266, 384

Koschack R., Rendtel J., 1990a, WGN, Journal of the International Meteor Organization, 18, 44

Koschack R., Rendtel J., 1990b, WGN, Journal of the International Meteor Organization, 18, 119

Kretschmer J., Drolshagen S., Koschny D., Drolshagen G., Poppe B., 2015, in Rault J.-L., Roggemans P., eds, Proceedings of the International Meteor Conference Mistelbach, Austria, 27-30 August 2015. pp 209213

Landgraf M., Baggaley W. J., Grün E., Krüger H., Linkert G., 2000, J. Geophys. Res., 105, 10343

Molau S., Barentsen G., 2013, in Gyssens M., Roggemans P., eds, Proceedings of the International Meteor Conference, 31st IMC, La Palma, Canary Islands, Spain, 2012. pp 11-17

Molau S., Barentsen G., 2014, Earth Moon and Planets, 112, 1

Olech A., 2000, WGN, Journal of the International Meteor Organization, 28,88

Ott T., Drolshagen E., Koschny D., Drolshagen G., Poppe B., 2014, in Rault J.-L., Roggemans P., eds, Proceedings of the International Meteor Conference, Giron, France, 18-21 September 2014. pp 23-29

Peterson G., 1999, Dynamics of Meteor Outburst and Satellite Mitigation Strategies. The Aerospace Press, El Segundo, CA
Rendtel J., Brown P., 1997, in Knofel A., ed., Proceedings of the International Meteor Conference, 15th IMC, Apeldoorn, Netherlands, 1996. pp 53-61 Suggs R. M., Moser D. E., Cooke W. J., Suggs R. J., 2014, Icarus, 238, 23 Weryk R. J., Brown P. G., 2013, Planet. Space Sci., 81, 32

Weryk R. J., Brown P. G., Domokos A., Edwards W. N., Krzeminski Z., Nudds S. H., Welch D. L., 2008, Earth Moon and Planets, 102, 241

This paper has been typeset from a $\mathrm{T}_{\mathrm{E}} \mathrm{X} / \mathrm{LAT} \mathrm{E} \mathrm{X}$ file prepared by the author. 